\title{
Análise da sintomatologia de mulheres climatéricas ativas e sedentárias
}

\author{
Analysis of the symptomatology of active and sedentary climacteric women \\ Análisis de la sintomatologia de mujeres climactericas activas y sedentarias
}

Recebido: 09/05/2021 | Revisado: 16/05/2021 | Aceito: 24/06/2021 | Publicado: 09/07/2021

Lucília da Costa Silva

ORCID: https://orcid.org/0000-0001-9386-5684 Centro Universitário Santo Agostinho, Brasil E-mail: luciliafisio@outlook.com

Ana Lys Marques Feitosa

ORCID: https://orcid.org/0000-0003-2004-6279 Centro Universitário UniFacid Wyden, Brasil E-mail: anallys@gmail.com

Kamylla Farias de Oliveira

ORCID: https://orcid.org/0000-0002-4610-2294

Universidade Estadual do Piauí, Brasil

E-mail: kamyllafariasoli20@gmail.com

Maria de Fátima de Carvalho Calaça ORCID: https://orcid.org/0000-0002-4263-0137

Christus Faculdade do Piauí, Brasil E-mail: carvalho-fatima@ outlook.com

Vandelma Lopes de Castro

ORCID: https://orcid.org/0000-0001-6822-2548

Universidade Estadual do Piauí, Brasil

E-mail: vandelmacastro@hotmail.com

Paulo Roberto Pereira Borges

ORCID: https://orcid.org/0000-0003-0541-7967

Universidade Estadual do Piauí, Brasil

E-mail: ppereiraborges@gmail.com

Anderson Lages Carvalho

ORCID: https://orcid.org/0000-0002-2109-5542

Associação de Ensino Superior do Piauí, Brasil

E-mail: andersonlages@hotmail.com Jehú Portela Sérvio

ORCID: https://orcid.org/0000-0003-3578-3642 Faculdade do Piauí, Brasil

E-mail: jehu.fisio@gmail.com

Samuel Lopes dos Santos

ORCID: https://orcid.org/0000-0003-3375-9171 Universidade Federal do Piaú, Brasil

E-mail: samuellopes121314@gmail.com

Francisca Taysa de Abreu Silva

ORCID: https://orcid.org/0000-0003-2081-9788

Centro Universitário Santo Agostinho, Brasil

E-mail: taysa.abreu163@gmail.com

Rayssa Stéfani Sousa Alves

ORCID: https://orcid.org/0000-0002-9666-675X

Pontifícia Universidade Católica de Góias, Brasil

E-mail: rayssastefani02@gmail.com

Fágner Magalhães

ORCID: https://orcid.org/0000-0001-8956-7115

Faculdade Maurício de Nassau, Brasil

E-mail:fagner.m.magalhães16@hotmail.com

Vitoria Vilas Boas da Silva Bomfim

ORCID: https://orcid.org/0000-0003-4897-0279

Centro Universitário Jorge Amado, Brasil

E-mail: pesquisaclinica9@gmail.com.

Larisse Passos Ribeiro Portela

ORCID: https://orcid.org/0000-0001-8883-084X

Centro Universitário Santo Agostinho, Brasil

E-mail:larisseportela@icloud.com

Antônio Lucas Farias da Silva

ORCID: https://orcid.org/0000-0001-8010-1714

Centro Universitário Unifacid, Brasi

E-mail: lucas1992farias@gmail.com 


\begin{abstract}
Resumo
Objetivos: Analisar a sintomatologia de mulheres climatéricas ativas e sedentárias. Métodos: A pesquisa transversal foi realizada com 60 mulheres climatéricas, no qual foram divididas em três grupos: 20 que desempenhava exercício aeróbico, 20 que realizava anaeróbico e 20 sedentárias. A coleta de dados foi realizada por meio de dois questionários referente às características sociodemográficas e pelo Menopause Rating Scale (MRS). Para análise dos dados, foi usado o teste Pearson Qui-quadrado com intervalo de confiança em 95\% e significância estabelecida em $p<0,05$. Resultados: A média de idade dos grupos foi $49,25 \pm 0,97$ anos a $52,95 \pm 1,37$ anos. Os resultados apontam uma diferença significativa para os grupos que praticam exercícios físicos com um declínio de sintomatologia climatéricas. Conclusão: O estudo mostrou, que as mulheres que exerciam exercícios anaeróbicos acompanhado por profissional, obtiveram um melhor resultado na diminuição dos sintomas climatéricos e melhor qualidade de vida.
\end{abstract}

Palavras-chave: Modalidades de fisioterapia; Saúde da mulher; Climatério; Exercícios físico.

\begin{abstract}
Objectives: To analyze the symptoms of active and sedentary climacteric women. Methods: The cross-sectional research was carried out with 60 climacteric women, where they were divided into three groups: 20 who played aerobic exercise, 20 performed anaerobic and 20 sedentary. Data collection was performed using two questionnaires related to sociodemographic characteristics and the Menopause Rating Scale (MRS). For data analysis, the Pearson Chi-square test was used with a 95\% confidence interval and significance set at $\mathrm{p}<0.05$. Results: The average age of the groups was $49.25 \pm 0.97$ years to $52.95 \pm 1.37$ years. The results point to a significant difference for the groups who practice physical exercises with a decline in climacteric symptoms. Conclusion: The study showed that women, who exercised anaerobic exercises accompanied by a professional, obtained a better result in the decrease of climacteric symptoms and a better quality of life.
\end{abstract}

Keywords: Physiotherapy modalities; Women's health; Climacteric; Physical exercise.

\begin{abstract}
Resumen
Objetivos: Analizar los síntomas de mujeres climatéricas activas y sedentarias. Métodos: La investigación transversal se realizó con 60 mujeres climatéricas, donde se dividieron en tres grupos: 20 que hacen ejercicio aeróbico, 20 que realizaban ejercicio anaeróbico y 20 sedentarias. La recolección de datos se realizó mediante dos cuestionarios relacionados con las características sociodemográficas y la Escala de Calificación de la Menopausia (MRS). Para el análisis de datos, se utilizó la prueba de chi-cuadrado de Pearson con un intervalo de confianza del 95\% y una significancia establecida en $\mathrm{p}<0,05$. Resultados: La edad promedio de los grupos fue de 49,25 $\pm 0,97$ años a 52,95 \pm 1,37 años. Los resultados apuntan a una diferencia significativa para los grupos que practican ejercicios físicos con una disminución de los síntomas climatéricos. Conclusión: El estudio mostró que las mujeres que ejercitado ejercicio anaeróbicos acompañadas de un profesional, obtienen un mejor resultado en la disminución de los síntomas climatéricos y una mejor calidad de vida.
\end{abstract}

Palabras clave: Modalidades de fisioterapia; La salud de la mujer; Climatérico; Ejercicio físico.

\title{
1. Introdução
}

O climatério é caracterizado pelo estado fisiológico do hipoestrogenismo, com a interrupção definitiva dos ciclos menstruais. Durante está nova fase de vida, a maioria das mulheres refere sintomas vasomotores, psicológicos e urogenitais, nos anos que seguem à menopausa, nos quais tendem a influenciar negativamente na qualidade de vida. Os sintomas, acometem entre 60 a $80 \%$ das mulheres e são reconhecidos como indutores de desconforto físico e emocional, que aumentam com a severidade dos sintomas (Daan et al. 2015; Portela et al., 2017).

A incidência de fatores de risco para doenças cardiovasculares e a mortalidade por todas as causas, são mais elevadas nas mulheres menopausadas em comparação com mulheres em idades reprodutivas. As terapias hormonais repositoras têm avançado na busca por sanar, na medida do possível, os problemas associados ao decréscimo de hormônios que ocorrem em função do climatério. Nesta direção a terapia baseada no uso de estrógenos passou recentemente a ser indicada com critérios mais rígidos, os quais buscam formas interativas do método com outras práticas paralelas, tais como adequação nutricional e aderência à prática de atividades físicas. Uma das alternativas não farmacológicas mais eficazes na redução dos sintomas climatéricos e na prevenção primária e secundária de inúmeras doenças crônicas é a prática de exercícios físicos regulares (Colpani et al., 2018; Lorenzi et al., 2009; Mandrup et al., 2018; Yu et al., 2019).

Ser fisicamente ativa tende a ter benefícios endógenos naturais, pois o exercício físico aumenta a liberação do hormônio adrenocorticotrófico (ACTH), e consequentemente, da adrenalina e dos corticoides, da testosterona, da prolactina, do 
hormônio do crescimento (GH) e das endorfinas, ao mesmo tempo em que diminui o hormônio luteinizante (LH), o hormônio folículo estimulante (FSH), aumentando os esteróides ovarianos e o hormônio estimulante da tireoide (TSH).As mulheres no período do climatério tornam um público que requer maior atenção e cuidado, existindo, portanto, a necessidade de uma melhor atuação por parte dos profissionais envolvidos com a saúde da mulher. Conhecer os fatores comprometedores da qualidade de vida das mulheres climatéricas é o ponto de partida para a formação de programas e estratégias de promoção à saúde (Wilmore et al., 2013; Serpa et al., 2016; Cabral et al., 2020). Diante do exposto, tivemos o objetivo de analisar a sintomatologia de mulheres climatéricas que desempenham exercícios anaeróbico, aeróbico e sedentárias.

\section{Metodologia}

O presente estudo, trata-se de uma pesquisa descritiva, intencional de corte transversal, analítica com abordagem quantitativa. Aprovado pelo Comitê de Ética em pesquisa do Centro Universitário Santo Agostinho(UNIFSA), sob parecer número 1.597.376, sendo desenvolvida conforme legislação específica, Resolução no 466/12 do Conselho Nacional de Saúde. A coleta de dados foi realizada no mês de setembro com 60 mulheres climatéricas, foi parcialmente realizada na Avenida Raul Lopes (Zona Leste de Teresina-PI), as sedentárias foram por meio de visitas a domicílio, já com as que exercia exercício anaeróbico foi realizado em um centro de academia em Teresina-PI. Foram divididas em três grupos: 20 que desempenhava exercício aeróbico, 20 que realizava fortalecimento muscular e 20 sedentárias. Em relação aos procedimentos, as participantes foram indagada pelos autores, àquelas que preenchiam os critérios de inclusão foram convidadas a participar da pesquisa, logo após foram informadas sobre: os objetivos do estudo, benefícios, riscos, posteriormente foi disponibilizado um Termo de Consentimento Livre e Esclarecido (TCLE) em duas vias de igual teor, que foi assinada, deixando comprovada sua participação.

Como critérios de exclusão: Mulheres climatérica que fazem reposição hormonal. Nos critérios de inclusão: se enquadrarão mulheres com no mínimo um ano de prática de exercício aeróbico (caminhada) ou treino anaeróbico (fortalecimento muscular), apresentando idade de 45 a 60 anos. Nos três grupos, foram realizadas as mesmas condutas, os dados sociodemográficos foram coletados através de um questionário específico, desenvolvido pelos autores. Para a avaliação dos sintomas do climatério e da qualidade de vida relacionada à saúde, utilizou-se o Menopause Rating Scale (MRS), o mesmo foi elaborado com caráter multidimensional, permitindo avaliar além da sintomatologia climatérica, a percepção geral de qualidade de vida em saúde. Este instrumento é específico, validado e reconhecido para uso no Brasil. A escala (MRS) é composta por 11 questões distribuídas por três domínios: somato vegetativos (fogachos, desconforto no coração, problemas com o sono e musculares e articulares), psicológicos (humor depressivo, irritabilidade, ansiedade, exaustão física e mental) e urogenitais (problemas de bexiga e sexuais e ressecamento da vagina). Cada sintoma pode ser classificado pela sua ausência e/ ou intensidade desse modo: $0=$ ausência, $1=$ leve, $2=$ moderado, $3=$ severo e $4=$ muito severo. A pontuação por domínios é realizada por meio do somatório dos referidos sintomas e a gravidade dos sintomas em cada domínio se dá da seguinte forma: ausente ou ocasional ( 0 a 4 pontos); leve ( 5 a 8 pontos); moderado ( 9 a 15 pontos); ou grave (16 pontos). Quanto maior a pontuação obtida, mais severa a sintomatologia e pior a qualidade de vida da mulher (Heinemann et al., 2003).

A análise estatística dos dados foi realizada de forma descritiva, utilizando o programa Microsoft Office Excel 2010, sendo distribuídos conforme os objetivos estabelecidos. Posteriormente os dados foram transferidos para o programa estatístico SpSS 20.0, no qual foram submetidos ao teste Pearson Qui-quadrado com intervalo de confiança em 95\% e significância estabelecidos em $\mathrm{p}<0,05$, nos resultados apresentamos em gráfico e tabelas. 


\section{Resultados}

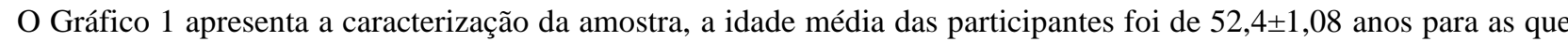

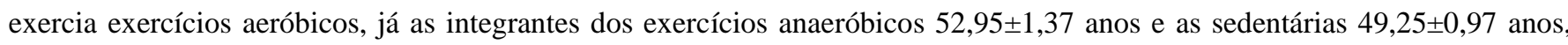
não foram verificadas diferenças significativas entre os três grupos.

Gráfico 1: Idades das participantes do estudo (praticantes de exercícios e sedentárias), Teresina-PI, 2021.

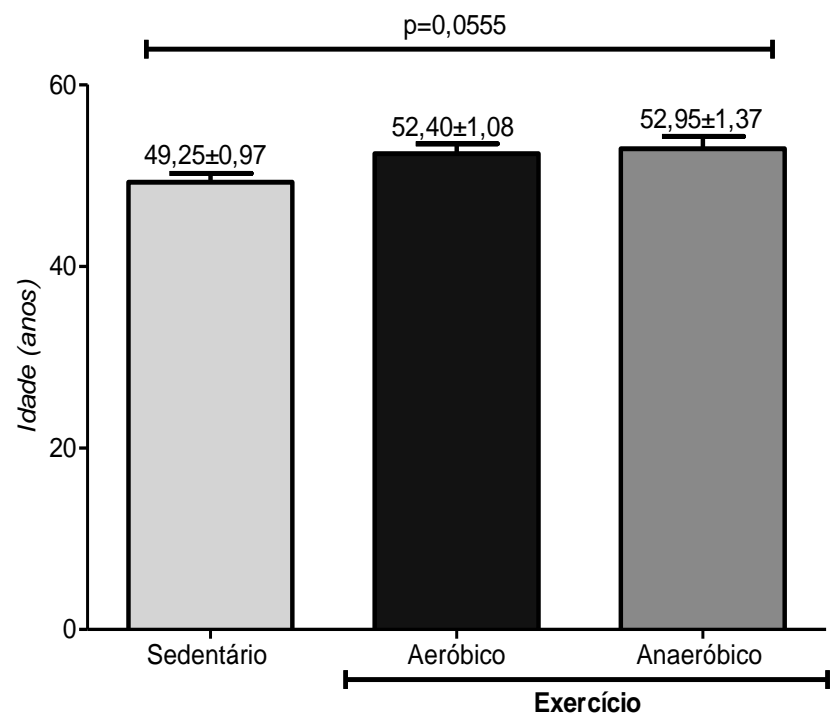

Fonte: Autores (2021).

Tabela 1: Distribuição das variáveis socioculturais pelas práticas de atividades praticadas, Teresina-PI, 2021.

\begin{tabular}{|c|c|c|c|c|c|c|c|}
\hline \multirow{3}{*}{ VARIÁVEIS } & \multicolumn{6}{|c|}{ Pratica de atividade } & \multirow{3}{*}{$\mathrm{P}$} \\
\hline & \multicolumn{2}{|c|}{ Aeróbico } & \multicolumn{2}{|c|}{ Anaeróbico } & \multicolumn{2}{|c|}{ Sedentárias } & \\
\hline & $\mathrm{n}$ & $\%$ & $\mathrm{n}$ & $\%$ & $\mathrm{~N}$ & $\%$ & \\
\hline \multicolumn{8}{|l|}{ Profissão } \\
\hline Profissionais ativos & 18 & $90,0 \%$ & 13 & $65,0 \%$ & 8 & $40,0 \%$ & \multirow[t]{2}{*}{$0,0030 * *$} \\
\hline Dona de casa & 2 & $10,0 \%$ & 7 & $35,0 \%$ & 12 & $60,0 \%$ & \\
\hline \multicolumn{8}{|l|}{ Patologia declaradas } \\
\hline Ansiedade & - & - & 2 & $10,0 \%$ & - & - & \multirow{12}{*}{0,1598} \\
\hline Arritmia cardíaca & - & - & 2 & $10,0 \%$ & - & - & \\
\hline Colesterol & - & - & 2 & $10,0 \%$ & - & - & \\
\hline Depressão & - & - & - & - & 2 & $10,0 \%$ & \\
\hline Diabetes, ansiedade e hipertensão & - & - & - & - & 1 & $5,0 \%$ & \\
\hline Diabetes, hipertensa & 1 & $5,0 \%$ & - & - & - & - & \\
\hline Hérnia de e disco & - & - & - & - & 1 & $5,0 \%$ & \\
\hline Hipertensa & 2 & $10,0 \%$ & - & - & - & - & \\
\hline Hipertensão & 2 & $10,0 \%$ & 2 & $10,0 \%$ & 2 & $10,0 \%$ & \\
\hline Hipertensão e ansiedade & 1 & $5,0 \%$ & - & - & - & - & \\
\hline Hipertensão e diabetes & - & - & 1 & $5,0 \%$ & - & - & \\
\hline Não & 14 & $70,0 \%$ & 11 & $55,0 \%$ & 14 & $70,0 \%$ & \\
\hline
\end{tabular}

Legenda: n, frequência absoluta; \%, frequência relativa; p para p teste de correlação de Pearson com IC 95\% e significância em $\mathrm{p}<0,05$.Fonte: Autores (2021). 
$\mathrm{Na}$ relação das profissões, podemos observar que as praticantes de atividade física dos dois grupos, tem um percentual de $90-65 \%$ das mulheres ativas profissionalmente, em comparação com as sedentárias que são $60 \%$ donas de casa. Nas patologias declaradas $70 \%$ das sedentárias, afirmam não possuir nenhuma patologia, já nos grupos das praticantes de exercícios físicos aeróbicos e anaeróbico apresentam 70\% e 55,0\% respectivamente.

Tabela 2: Compreensão da Menopause Rating Scale (MRS) e os seu respectivos escores, Teresina-PI, 2021.

\begin{tabular}{|c|c|c|c|c|c|c|c|}
\hline \multirow{3}{*}{ VARIÁVEIS } & \multicolumn{6}{|c|}{ Pratica de atividade } & \multirow{3}{*}{$\mathrm{p}$} \\
\hline & \multicolumn{2}{|c|}{ Aeróbico } & \multicolumn{2}{|c|}{ Anaeróbico } & \multicolumn{2}{|c|}{ Sedentárias } & \\
\hline & $\mathrm{n}$ & $\%$ & $\mathrm{n}$ & $\%$ & $\mathrm{n}$ & $\%$ & \\
\hline \multicolumn{8}{|l|}{ Qualidade do sono } \\
\hline Bom & 5 & $23,8 \%$ & 3 & $15,0 \%$ & 1 & $5,0 \%$ & \multirow{3}{*}{0,1467} \\
\hline Presença de Distúrbio do Sono & 3 & $14,3 \%$ & 9 & $45,0 \%$ & 7 & $35,0 \%$ & \\
\hline Ruim & 13 & $61,9 \%$ & 8 & $40,0 \%$ & 12 & $60,0 \%$ & \\
\hline \multicolumn{8}{|l|}{ Sintomas somato-vegetativos } \\
\hline Assintomáticos ou escassos & 7 & $33,3 \%$ & 4 & $20,0 \%$ & 2 & $10,0 \%$ & \multirow{5}{*}{$0,0015^{* *}$} \\
\hline Escassos & 0 & $0,0 \%$ & 6 & $30,0 \%$ & 0 & $0,0 \%$ & \\
\hline Leves & 6 & $28,6 \%$ & 7 & $35,0 \%$ & 5 & $25,0 \%$ & \\
\hline Moderados & 8 & $38,1 \%$ & 2 & $10,0 \%$ & 9 & $45,0 \%$ & \\
\hline Severos & 0 & $0,0 \%$ & 1 & $5,0 \%$ & 4 & $20,0 \%$ & \\
\hline \multicolumn{8}{|l|}{ Sintomas psicológicos } \\
\hline Assintomáticos ou escassos & 7 & $33,3 \%$ & 2 & $10,0 \%$ & 3 & $15,0 \%$ & \multirow{5}{*}{$0,0160^{*}$} \\
\hline Escassos & 0 & $0,0 \%$ & 6 & $30,0 \%$ & 0 & $0,0 \%$ & \\
\hline Leves & 3 & $14,3 \%$ & 5 & $25,0 \%$ & 3 & $15,0 \%$ & \\
\hline Moderados & 5 & $23,8 \%$ & 4 & $20,0 \%$ & 7 & $35,0 \%$ & \\
\hline Severos & 6 & $28,6 \%$ & 3 & $15,0 \%$ & 7 & $35,0 \%$ & \\
\hline \multicolumn{8}{|l|}{ Sintomas urogenitais } \\
\hline Assintomáticos ou escassos & 7 & $33,3 \%$ & 2 & $10,0 \%$ & 4 & $20,0 \%$ & \multirow{5}{*}{$0,0062 * *$} \\
\hline Escassos & 0 & $0,0 \%$ & 8 & $40,0 \%$ & 0 & $0,0 \%$ & \\
\hline Leves & 2 & $9,5 \%$ & 2 & $10,0 \%$ & 4 & $20,0 \%$ & \\
\hline Moderados & 5 & $23,8 \%$ & 3 & $15,0 \%$ & 6 & $30,0 \%$ & \\
\hline Severos & 7 & $33,3 \%$ & 5 & $25,0 \%$ & 6 & $30,0 \%$ & \\
\hline \multicolumn{8}{|l|}{ Classificação } \\
\hline Assintomáticos ou escassos & 7 & $33,3 \%$ & 10 & $50,0 \%$ & 1 & $5,0 \%$ & \multirow{4}{*}{$0,0045^{* *}$} \\
\hline Leves & 2 & $9,5 \%$ & 4 & $20,0 \%$ & 6 & $30,0 \%$ & \\
\hline Moderados & 10 & $47,6 \%$ & 2 & $10,0 \%$ & 5 & $25,0 \%$ & \\
\hline Severos & 2 & $9,5 \%$ & 4 & $20,0 \%$ & 8 & $40,0 \%$ & \\
\hline
\end{tabular}

Legenda: n, frequência absoluta; \%, frequência relativa; p para p teste de correlação de Pearson com IC 95\% e significância em $\mathrm{p}<0,05$. Fonte: Autores (2021).

Na (Tabela 2) por meio da análise da Menopause Rating Scale (MRS), cuja escala avalia o grau de severidade da sintomatologia no climatério, observou-se que no grupo anaeróbico apresentou $40 \%$ em relação ao sono ruim, o mesmo grupo em relação aos distúrbios do sono apresentam uma porcentagem superior aos outros grupos estudados com $45 \%$, já as praticantes de exercícios aeróbico destacam $61 \%$ com presença de sono ruim. No grupo das sedentárias observou-se uma porcentagem de $5 \%$ de sono bom e $60 \%$ sono ruim, em relação às variáveis, não houve diferença significante. Entretanto entre os domínios somato-vegetativos, o grupo com um grau de severidade mais acentuado foram às sedentárias que de acordo com 
o estudo apresentou $45 \%$ moderados e $20 \%$ severos, em comparação com as mulheres que praticam exercícios aeróbicos sintomatologia moderada $38,1 \%$ e severa $0 \%$, já a que destacou um melhor resultado nesse foram as praticantes de exercícios anaeróbicos que apenas $10 \%$ moderados e $5 \%$ severos e com $50 \%$ das participantes assintomáticas.

Nas subescalas que prosseguem sintomas psicológicos e urogenitais, podemos observar mais uma vez uma diferença das mulheres praticantes de exercícios anaeróbicos, no domínio Psicológicos 33,3\% delas são assintomáticas, em contra partida somente $15 \%$ das sedentárias são assintomáticas e 28,6\% possuem sintomas severos, já no domínio de sintomas urogenitais $50 \%$ das praticantes de exercícios anaeróbico são assintomáticas e apenas $20 \%$ e 33,3\% das sedentárias e praticantes de exercícios aeróbicos respectivamente são assintomáticas. Na classificação geral, as mulheres que realizava exercícios anaeróbicos 50\% da amostra relata ser assintomáticas, em comparação apenas 5\% das sedentárias e 33,3\% das que desempenhava exercícios aeróbicos, relatam ser assintomáticas.

\section{Discussão}

A pesquisa destaca os exercícios físicos, como um meio não farmacológica capaz de ocasionar um declínio na sintomatologia das mulheres no climatério. Os resultados encontrados mostram que as mulheres que exercem exercícios físicos sobressaem sobre as sedentárias, porém o grupo com maior relevância de acordo coma análise do questionário (MRS), foi o grupo das mulheres que desempenhava exercícios anaeróbicos.

O impacto do sedentarismo no aumento das queixas relativas aos sintomas climatéricos e na incidência de doenças crônicas não transmissíveis se reflete negativamente na qualidade de vida das mulheres menopausadas (Gonçalves et al.2011). Em uma outra pesquisa, as mulheres praticantes de exercícios físicos, apresentaram menor intensidade em todos os sintomas climatéricos, em comparação com as que não praticavam exercícios físicos (Barreto et al., 2015). Na observação encontrada por Tairova et al. (2011) em seu estudo, com o objetivo de avaliar a qualidade de vida e sintomatologia de mulheres no climatério, analisaram 197 mulheres, divididas em dois grupos, sedentárias e praticantes de exercícios aeróbicos, mostraram que as praticantes de exercício sobressaem diante das sedentárias, corroborando com o presente estudo afirmando que os exercícios físicos são bastante efetivos para a diminuição dos sintomas vasomotores, como os fogachos e sudorese noturna. Segundo Boecker et al. (2008), o exercício físico pode aumentar a produção de beta-endorfinas e estimular a atividade central de opioides, diminuindo assim os fogachos.

Um estudo que comparou 2.204 mulheres menos ativas com as mais ativas, observou que a prática de atividades físicas esteve relacionada com a melhora dos sintomas climatéricos, dentre eles os de natureza psicológica (Kim et al.,2014). Na pesquisa de base populacional realizado em Natal/RN com 370 mulheres de 40 a 65 anos, mostrou também que as mulheres ativas relataram menor intensidade e menor prevalência de sintomas climatéricos quando comparadas às sedentárias (Canário et al. 2012; Probo et al 2016). Zanesco et al. (2009), observou no seu estudo e destaca que exercício aeróbico (como meia hora de caminhada, por exemplo) proporciona melhorias significativas de alguns sintomas do climatério, dentre eles, transtorno de ansiedade e a depressão.

Em Portugal, Guimarães e Baptista (2009), realizaram um estudo com 104 mulheres de meia idade, agrupadas de acordo com a duração da sessão diária de atividade física que realizavam (grupo A - $30 \mathrm{~min} / \mathrm{dia}$; grupo B - 30-60 min/dia; grupo C - $60 \mathrm{~min} / \mathrm{dia}$ ). Ao final de 12 semanas de intervenção foi constatado que as mulheres com prática de atividade física de $60 \mathrm{~min} /$ dia mostraram diminuição da intensidade dos sintomas climatérios, bem como melhor percepção dos domínios psicológico e social da qualidade de vida. Estes dados sugerem que a adoção da prática regular de atividades físicas moderadas pode exercer efeitos positivos significativos na saúde da mulher climatérica.

A prática regular de exercícios físicos é uma opção terapêutica de baixo custo e com boas efetividades, para diminuir os sintomas e melhorar a qualidade de vida de mulheres no período do climatério. Com base, nesse estudo o referido trabalho 
também apresentou queixas subjetivas em todos os grupos, entretanto os resultados desta pesquisa devem ser interpretados de acordo com suas limitações. Os sintomas climatéricos foram avaliados por questionário validado, sem terem sido considerados diagnósticos clínicos.

Corroborando, estudos afirmam que questionários de auto aplicação que apresentem alto grau de confiabilidade validade e fidedignidade podem ser os instrumentos mais adequados, pois estes são capazes de avaliar aspectos subjetivos envolvidos com a sintomatologia climatérica (Cabral 2013). Diante disso, pesquisas que abordem a relação entre a sintomatologia climatérica, ainda são escassos na literatura vigente.

\section{Conclusão}

Portanto, conclui-se que os exercícios físicos anaeróbicos, acompanhados por profissional qualificado e apoio especializado, apresentam uma maior efetividade na melhora da sintomatologia e qualidade de vida. É de suma importância à realização de novos estudos voltados para o período climatérico, espera-se que os achados apresentados nessa pesquisa venham contribuir, para a promoção de saúde de mulheres climatéricas, valorizando a prática regular de exercícios físicos.

\section{Referências}

Barreto, H. V., A, Alves, T. T. M, Soares, N. I. S, Silva, V. F, \& Cabral, P. U. L. (2015). Atividade física na saúde e qualidade de vida de mulheres climatéricas. Cinergis, 16(3):203-208.

Boecker, H., Sprenger, T., Spilker, M. E., Henriksen, G., Koppenhoefer, M., Wagner, K. J., \& Tolle, T. R. (2008).The runner's high: opioidergic mechanisms in the human brain. Cereb Cortex, 18(11):2523-31.

Canário, A. C., Cabral, P. U., Spyrides, M. H., Giraldo, P. C., Eleutério Jr, J., \& Gonçalves, A. K. (2012)The impact of physical activity on menopausal symptoms in middle-aged women. Int J Gynaecol Obstet, 118(1):34-6.

Cabral P. U. L, Canário A. C, Spyrides M. H, Uchôa A. S Júnior, E. J, \& Gonçalves, A. K. (2013). Determinants of sexual dysfunction among middle-aged women. International J Gynaecol Obstet, 120(3):271-4.

Cabral, P. U. L., Carvalho, B. E., Silva, M. S., Spíndola, P. S., Silva, M. C. B., Soares, N. I. S., et al(2020). Nível de atividade física, sintomas climatéricos e qualidade de vida relacionada à saúde em mulheres na pós-menopausa. Rev Bras Fisiol Exerc,19(3):192- 201.

Colpani, V., Baena, C. P., Jaspers, L., Van Dijk, G. M., Farajzadegan, Z., Dhana, K., \& Franco, O. H. (2018). Lifestyle factors, cardiovascular disease and allcause mortality in middle-aged and elderly women: a systematic review and meta-analysis. European journal of epidemiology, 33(9), 831-845

Daan, N. M., \& Fauser, B. C. (2015). Menopause prediction and potential implications. Maturitas, 82(3), 257-265.

Gonçalves, A. K. D. S., Canário, A. C. G., Cabral, P. U. L., Silva, R. A. H. D., Spyrides, M. H. C., Giraldo, P. C., \& Eleutério Jr, J. (2011). Impacto da atividade física na qualidade de vida de mulheres de meia idade: estudo de base populacional. Revista brasileira de ginecologia e obstetrícia, 33 (12), 408-413.

Guimarães A.A.C, Baptista F. Influence of habitual physical activity on the symptoms of climacterium/menopause and the quality of life of middle-aged women. (2011). International journal of women's health, 3(1): 319,-328.

Heinemann L. A, Potthoff P, Schneider H. P. (2003). International versions of the menopause rating scale (MRS). Health and quality of life outcomes, 1(1):14.

Lorenzi, D. R. S. D., Catan, L. B., Moreira, K., \& Ártico, G. R. (2009). Assistência à mulher climatérica: novos paradigmas. Revista Brasileira de Enfermagem, 62(2), 287-293.

Mandrup, C. M., Egelund, J., Nyberg, M., Enevoldsen, L. H., Kjær, A., Clemmensen, A. E., \& Stallknecht, B. M. (2018). Effects of menopause and highintensity training on insulin sensitivity and muscle metabolism. Menopause, 25(2), 165-175.

Kim, M. J., Cho, J., Ahn, Y., Yim, G., \& Park, H. Y. (2014). Association between physical activity and menopausal symptoms in perimenopausal women. BMC women's health, 14(1), 1-8.

Probo, A., Soares, N., Silva, V., \& Cabral, P. (2016). Níveis dos sintomas climatéricos em mulheres fisicamente ativas e insuficientemente ativas. Revista Brasileira de Atividade Física \& Saúde, 21(3), 246-254.

Portela, L. P. R., Costa Silva, L., Rodrigues, T. F., de Oliveira, L. M. N., \& Mazullo Filho, J. B. R. (2017). Análise da qualidade do sono em Mulheres climatéricas ativas e sedentárias. ConScientiae Saúde, 16(2), 274-280.

Serpa, M. A., Lima, A. A., Guimarães, A. C. P., Carrilo, M. R. G. G., Coura-Vital, W., \& Veloso, V. M. (2016). Fatores associados à qualidade de vida em mulheres no climatério. Reprodução \& Climatério, 31(2), 76-81. 
Research, Society and Development, v. 10, n. 8, e15410815926, 2021

(CC BY 4.0) | ISSN 2525-3409 | DOI: http://dx.doi.org/10.33448/rsd-v10i8.15926

Tairova, O. S., \& Lorenzi, D. R. S. (2011). Influência do exercício físico na qualidade de vida de mulheres na pós-menopausa: um estudo casocontrole. Revista brasileira de geriatria e gerontologia, 14(1), 135-145.

Wilmore J, Costill DL. Fisiologia do esporte e do exercício. (5a ed.), Manole, 2013.

Yu, P. A., Hsu, W. H., Hsu, W. B., Kuo, L. T., Lin, Z. R., Shen, W. J., \& Hsu, R. W. W. (2019). The effects of high impact exercise intervention on bone mineral density, physical fitness, and quality of life in postmenopausal women with osteopenia: A retrospective cohort study. Medicine, 98(11).

Zanesco, A., \& Zaros, P. R. (2009). Exercício físico e menopausa. Revista Brasileira de Ginecologia e Obstetrícia, 31(5), 254-261. 